Quim. Nova, Vol. 35, No. 1, 30-34, 2012

\title{
FOTOELETRODEGRADAÇÃO DO CORANTE AO7 UTILIZANDO FILMES DE NANOCOMPÓSITOS DE $\mathrm{ZnO}_{-\mathrm{TiO}_{2}}$
}

\author{
Tânia Frade, Anabela Gomes* e Maria Isabel da Silva Pereira \\ Departamento de Química e Bioquímica, Faculdade de Ciências, Universidade de Lisboa, 1749-016 Lisboa, Portugal \\ Ana Lopes e Lurdes Ciríaco \\ Departamento de Química, Universidade da Beira Interior, 6201-001 Covilhã, Portugal
}

Recebido em 27/11/10; aceito em 18/5/11; publicado na web em 8/7/11

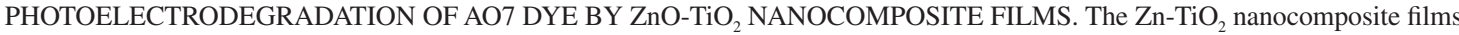
were prepared by electrodeposition, using an acidic zinc sulphate solution with $\mathrm{TiO}_{2}$ nanoparticles in suspension. The as-deposited samples have been heated in air at $450{ }^{\circ} \mathrm{C}$ for $6 \mathrm{~h}$. The XRD and SEM analyses pointed out to the metal matrix conversion from $\mathrm{Zn}$ to $\mathrm{ZnO}$ and a rich morphology of needles-shaped grains. These materials were used on the photoelectrochemical degradation of AO7, which was efficiently degraded, with $40 \%$ of color removal, after $2 \mathrm{~h}$ period at $1.0 \mathrm{~V}$, under white light irradiation. The apparent first order rate constant of the photoelectrodegradation reaction was $4.12 \times 10^{-3} \mathrm{~min}^{-1}$.
\end{abstract}

Keywords: $\mathrm{ZnO}-\mathrm{TiO}_{2}$ nanocomposite films; $\mathrm{AO} 7$ dye; photoelectrodegradation.

\section{INTRODUÇÃO}

Atualmente, os corantes utilizados na indústria têxtil exigem especial atenção, uma vez que a sua persistência no meio hídrico após o tratamento dos efluentes tem provocado problemas ambientais a nível mundial. ${ }^{1-3}$ Assim, o desenvolvimento de tecnologias eficazes para o tratamento de efluentes tem sido alvo de muita investigação. Existem vários métodos que permitem a remoção de cor e carga orgânica de efluentes que contenham corantes. No entanto, muitos destes métodos têm a desvantagem de serem de elevado custo, o que impossibilita a sua aplicação em larga escala na indústria têxtil. ${ }^{4}$

Os processos oxidativos avançados (POAs) mostram-se muito promissores no tratamento de efluentes, uma vez que demonstraram ser eficazes na degradação de poluentes orgânicos em águas e solos, sendo possível uma degradação quase total dos compostos. ${ }^{5}$ Os POAs utilizam o radical hidroxila $\left(\mathrm{HO}^{*}\right)$, que tem um elevado poder oxidante e pode promover a degradação de vários compostos poluentes num tempo relativamente reduzido. De modo geral, estes processos, envolvem oxidação química, ${ }^{6-8}$ eletroquímica, ${ }^{9}$ fotocatalítica ${ }^{10,11} \mathrm{e} / \mathrm{ou}$ fotoeletrocatalítica. ${ }^{12,13}$

Os processos de oxidação fotoeletrocatalítica têm sido a grande aposta por parte dos investigadores, pois têm demonstrado ser eficientes, econômicos e "amigos" do ambiente em várias aplicações industriais, ${ }^{14}$ incluindo a mineralização de poluentes orgânicos, ${ }^{15-17}$ purificação de água ${ }^{18}$ e ar, ${ }^{19}$ assim como na produção de combustíveis $^{20,21}$ e eletricidade. ${ }^{22}$ Este tipo de processo baseia-se, de modo geral, na irradiação de um material semicondutor por fotóns, suficientemente energéticos, que permitam a passagem de elétrons da banda de valência para a banda de condução $\left(e_{\mathrm{BC}}^{-}\right)$, criando lacunas carregadas positivamente $\left(h^{+}{ }_{\mathrm{BV}}\right)$ na banda de valência. ${ }^{23-25}$ Desta forma, os compostos orgânicos poderão ser degradados pelos radicais hidroxila formados através da reação da lacuna fotogerada com a água adsorvida à superfície do semicondutor ${ }^{26} \mathrm{e} / \mathrm{ou}$ da reação dos elétrons fotoinjetados com o $\mathrm{O}_{2}$ que formará um ânion radical superóxida, que irá sofrer reações sucessivas até a formação de radicais hidroxila. ${ }^{24,27}$ A aplicação de uma densidade de corrente ou de um potencial anódico constante ao fotoânodo, através de um circuito externo, irá

\footnotetext{
*e-mail: abmg@fc.ul.pt
}

permitir a fotogeração de elétrons que serão continuamente extraídos do fotoânodo, melhorando a eficiência do processo. ${ }^{23}$

Os semicondutores $\mathrm{TiO}_{2}$ e $\mathrm{ZnO}$ têm sido extensivamente estudados como fotocatalisadores ${ }^{15-19,28}$ e fotoeletrocatalisadores ${ }^{29-31}$ na degradação de poluentes orgânicos, devido ao seu baixo custo, alta eficiência, baixa toxicidade e alta estabilidade em meio aquoso. $\mathrm{O}$ problema da recombinação do par $e_{\mathrm{BC}}^{-} / h^{+}{ }_{\mathrm{BV}}$ gerado durante os processos fotocatalíticos é ultrapassado nos processos fotoeletrocatalíticos, pois aqui se induz o aumento do tempo de vida do par $e_{\mathrm{BC}}^{-} / h^{+}{ }_{\mathrm{BV}}$, com o consequente aumento da eficiência. ${ }^{32}$ Com o objetivo de ultrapassar este problema e expandir a região de absorção da luz, melhorando a eficiência dos processos, têm sido estudadas diferentes formas de preparar nanocompósitos de $\mathrm{ZnO}-\mathrm{TiO}_{2}{ }^{33}$

Existem vários métodos de preparação de nanocompósitos de $\mathrm{ZnO}-\mathrm{TiO}_{2}$, referenciados na literatura, sendo de salientar a oxidação térmica da matriz metálica por ser um método simples e econômico, permitindo produzir óxidos metálicos nanoestruturados e com dimensões diversas, mediante as condições de aquecimento. ${ }^{34}$

Neste trabalho prepararam-se, através de oxidação térmica, na-

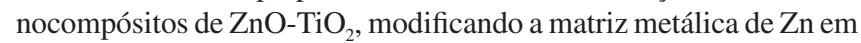
$\mathrm{ZnO}$, de modo a serem aplicados em fotoeletrocatálise.

O corante Acid Orange 7, AO7, foi escolhido como modelo por ser uma molécula simples e amplamente utilizado para coloração na indústria do papel e têxtil. ${ }^{35}$

\section{PARTE EXPERIMENTAL}

\section{Preparação dos nanocompósitos de $\mathrm{Zn}-\mathrm{TiO}_{2}$ e tratamento térmico}

A solução eletrolítica era composta por $0,1 \mathrm{~mol} \mathrm{dm}^{-3} \mathrm{ZnSO}_{4} \cdot 7 \mathrm{H}_{2} \mathrm{O}$ (Sigma-Aldrich, 99\%), 0,2 mol dm ${ }^{-3} \mathrm{MgSO}_{4} \cdot 7 \mathrm{H}_{2} \mathrm{O}$ (Sigma-Aldrich, $\geq$ 99\%), 0,15 mol dm ${ }^{-3} \mathrm{H}_{3} \mathrm{BO}_{3}$ (Panreac, 99.8\%) e $10 \mathrm{~g} \mathrm{dm}^{-3}$ de partículas de $\mathrm{TiO}_{2}\left(\right.$ Aeroxide $^{\circledR} \mathrm{P} 25$ ), sendo acidificada a $\mathrm{pH} 4$ pela adição de $\mathrm{H}_{2} \mathrm{SO}_{4}$ diluído. Todas as soluções foram preparadas diariamente sem purificação prévia, sendo desaeradas com fluxo de nitrogênio antes e durante a eletrodeposição.

Usou-se uma célula de vidro de dois compartimentos, com uma placa de $\mathrm{Zn}$ como eletrodo secundário e um eletrodo de $\mathrm{Ag} / \mathrm{AgCl}$ 
como referência. O eletrodo de trabalho era um disco de Ti com 0,8 $\mathrm{cm}^{2}$ de área (Goodfellow Corporation). O disco de Ti foi mergulhado em $\mathrm{HF}$ a $40 \%$, polido com sílica em pó de $0,05 \mu \mathrm{m}$ (Buehler) e limpo em ultrassom durante 10 min em água ultrapura Millipore Milli-Q.

Os nanocompósitos de $\mathrm{Zn}-\mathrm{TiO}_{2}$ foram preparados por eletrodeposição galvanostática pulsada, onde $i_{\mathrm{c}}$ e $i_{\mathrm{a}}$ são as densidades de corrente catódica e anódica (com os valores -19 e 19 mA cm${ }^{-2}$, respectivamente) e, $t_{\mathrm{c}}$ e $t_{\mathrm{a}}$ as durações de impulso catódico e anódico (com os valores 16 e $8 \mathrm{~ms}$, respectivamente).

A deposição foi realizada sob agitação $(150 \mathrm{rpm})$ à temperatura ambiente durante 100 min. Após terminada a deposição, o eletrodo foi retirado da célula, lavado com água ultrapura Millipore Milli-Q e seco sob fluxo de nitrogênio durante 5 a $10 \mathrm{~min}$.

As eletrodeposições foram realizadas usando um potenciostato/ galvanostato Autolab PGSTAT12, com aquisição automática de dados por software GPES 4.9.

Os nanocompósitos de $\mathrm{Zn}-\mathrm{TiO}_{2}$ foram posteriormente aquecidos ao ar durante $6 \mathrm{~h} \mathrm{a} 450^{\circ} \mathrm{C}$, usando uma velocidade de aquecimento de $1{ }^{\circ} \mathrm{C} /$ min. Para tal, utilizou-se um forno convencial Naberterm P320 controlado pelo programador Logotherm Controller S19.

\section{Caracterização dos filmes}

A caracterização estrutural dos nanocompósitos foi realizada por difração de raios-X (DRX), num difratómetro Philips Analytical PW 3050/60 X'Pert PRO equipado com um detetor X'Celerator e com aquisição automática de dados através do software X'Pert Data Collector (V2.0b), usando uma radiação monocromática de $\mathrm{CuK}_{\alpha}$ como feixe incidente, operando a $40 \mathrm{kV}$ e $30 \mathrm{~mA}$.

Os difratogramas foram obtidos por varrimento contínuo num intervalo de $2 \theta$ de 20 a $90^{\circ}$, com um tamanho de passo $2 \theta$ de $0,02^{\circ} \mathrm{e}$ um tempo de aquisição de $10 \mathrm{~s} /$ passo.

A caracterização morfológica e elementar dos filmes foi realizada por microscopia eletrônica de varredura (FEG-MEV Jeol 7001F) e microanálise semiquantitativa por dispersão de energias (EDS), com um feixe de elétrons de $25 \mathrm{kV}$ de potência.

\section{Ensaios de fotoeletrodegradação}

Os ensaios de fotoeletrodegradação do corante AO7 foram efetuados usando uma solução aquosa de $0,035 \mathrm{~mol} \mathrm{dm}^{-3} \mathrm{Na}_{2} \mathrm{SO}_{4}$ (pH 6,2) como eletrólito suporte, com 5 ppm do sal de sódio 4-dimetilaminoazobenzeno-4'-sulfónico, conhecido como Acid Orange 7 (AO7) (Sigma-Adrich).

Os ensaios foram realizados potenciostaticamente, sendo o valor de potencial aplicado de 0,8 e $1,0 \mathrm{~V}$ vs $\mathrm{Ag} / \mathrm{AgCl}$, durante $2 \mathrm{~h}$, sob agitação, à temperatura ambiente. Os eletrodos de trabalho foram iluminados, através de uma janela de quartzo, a $365 \mathrm{~nm}$ e com luz branca colocada a $16 \mathrm{~cm}$ de distância. O volume de solução foi $100 \mathrm{~mL}$.

O processo de fotoeletrodegradação foi acompanhado por espetroscopia de UV-Vis, num intervalo de 200 a $700 \mathrm{~nm}$ através de um espetrofotómetro Jasco V-560, com aquisição automática de dados via software Jasco Spectra Manager (V1.52). Utilizaram-se células de quartzo de $1 \mathrm{~cm}$ de percurso ótico. A concentração do AO7 foi determinada através dos valores de absorvância a $\lambda=485$ $\mathrm{nm}$ e a percentagem de remoção da cor foi calculada através da Equação 1:

$$
\text { Percentagem de remoção }=\frac{A b s_{0}-A b s}{A b s_{0}} \times 100 \%
$$

onde $A b s_{0}$ é o valor de absorvância da solução inicial. Fizeram-se recolhas regulares de solução em intervalos de 30 min para determinação da absorvância da solução $(A b s)$.

\section{RESULTADOS E DISCUSSÃO}

\section{Caracterização estrutural}

A identificação das fases constituintes dos nanocompósitos de $\mathrm{Zn}-\mathrm{TiO}_{2}$ antes e depois de sujeitos ao tratamento térmico foi feita com base nos difratogramas respectivos. Na Figura 1 apresenta-se o resultado dessa identificação.

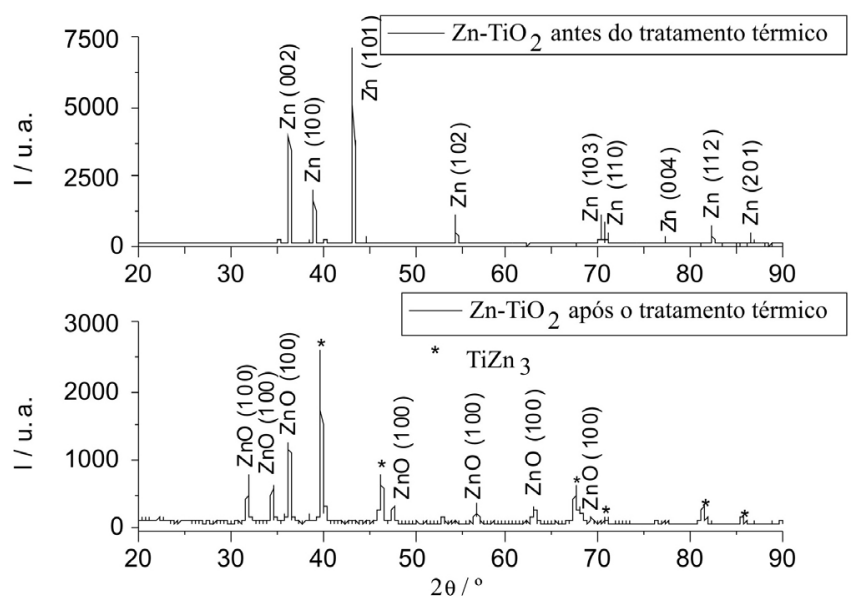

Figura 1. Difratogramas dos nanocompósitos de $\mathrm{Zn}-\mathrm{TiO}_{2}$ antes e após o tratamento térmico

Pode verificar-se que as reflexões antes do tratamento térmico correspondem à fase cristalina do $\mathrm{Zn}$ hexagonal. ${ }^{36}$ Após o aquecimento, o $\mathrm{Zn}$ da matriz metálica transforma-se, com sucesso, na estrutura cristalina de $\mathrm{ZnO}$ hexagonal. ${ }^{37}$ Este fato poderá ser atribuído à presença das partículas de $\mathrm{TiO}_{2}$ incorporadas na matriz metálica. Estudos realizados anteriormente comprovaram que a presença de nanopartículas incorporadas numa matriz metálica promove a diminuição do tamanho de cristalite $^{38}$ que, por sua vez, irá permitir uma conversão de uma matriz de $\mathrm{Zn}$ a $\mathrm{ZnO}$ bem sucedida. ${ }^{39,40}$ Segundo Lin et al. ${ }^{40}$ a presença das partículas de $\mathrm{TiO}_{2}$ afeta o crescimento do tamanho de grão da matriz metálica, impedindo o aumento deste durante o processo de recristalização por ação do aquecimento.

\section{Caracterização morfológica}

A morfologia da superfície dos nancompósitos de $\mathrm{Zn}-\mathrm{TiO}_{2}$ antes e depois do tratamento térmico é mostrada na Figura 2. Nas micrografias dos nanocompósitos de $\mathrm{Zn}-\mathrm{TiO}_{2}$ antes de aquecidos (Figura 2a), é visível um tamanho de grão de Zn reduzido e uma superfície morfologicamente irregular, com a formação de aglomerados. Após o aquecimento (Figura 2b), visualiza-se a formação de cristais hexagonais muito pequenos, nos quais crescem nanoagulhas com $\sim 1 \mu \mathrm{m}$ de altura, compostas por $\mathrm{Zn}$ e O, que foram identificados por EDS. Estes resultados estão de acordo com os reportados por Deguchi et al., ${ }^{39}$ que descrevem as condições otimizadas para se obter amostras de $\mathrm{Zn}-\mathrm{TiO}_{2}$ em aço, bem como as atividades fotocatalíticas após tratamento térmico. Aal et al. ${ }^{41}$ também provaram ser possível obter o mesmo tipo de morfologia na preparação de filmes de $\mathrm{ZnO}$ a $450{ }^{\circ} \mathrm{C}$.

De modo a interpretar o mecanismo de crescimento das nanoestruturas unidimensionais (1D) de $\mathrm{ZnO}$, podem ser considerados diferentes mecanismos de crescimento de cristais. A morfologia das nanoestruturas pode ser influenciada por vários parâmetros experimentais, tais como temperatura de reação, tamanho de grão e textura 

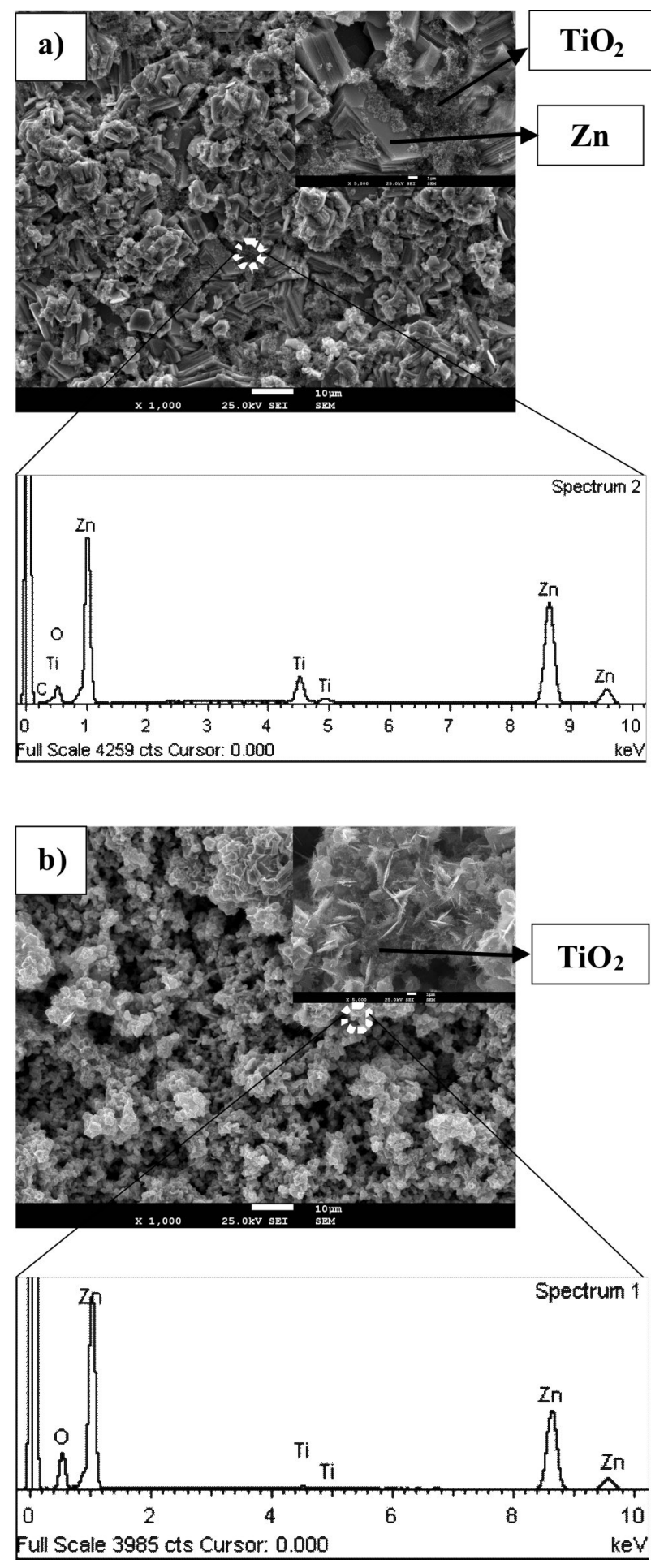

Figura 2. Micrografias e análise pontual por microanálise por energia dispersiva (EDS) dos nanocompósitos de $\mathrm{Zn}-\mathrm{TiO}_{2}$, antes (a) e depois (b) do tratamento térmico. Ampliações: imagens de SEM demonstrando a presença das partículas de $\mathrm{TiO}_{2}$ antes e depois do aquecimento

cristalográfica do $\mathrm{Zn}$ e velocidade de oxidação que favorecem o crescimento na direção axial e a inibição nas direções restantes. ${ }^{34,42-44}$

\section{Eletrodegradação fotoassistida do AO7}

A Figura 3 mostra a evolução dos espectros de UV-Vis durante os processos de fotoeletrodegradação realizados a potencial constante igual a $0,8 \mathrm{~V}$ vs $\mathrm{Ag} / \mathrm{AgCl}$, utilizando como fotoânodo um nanocompósito de $\mathrm{ZnO}-\mathrm{TiO}_{2}$ obtido por oxidação térmica, iluminado com luz monocromática $\lambda=365 \mathrm{~nm}$ (Figura 3a) e luz branca (Figura 3b).
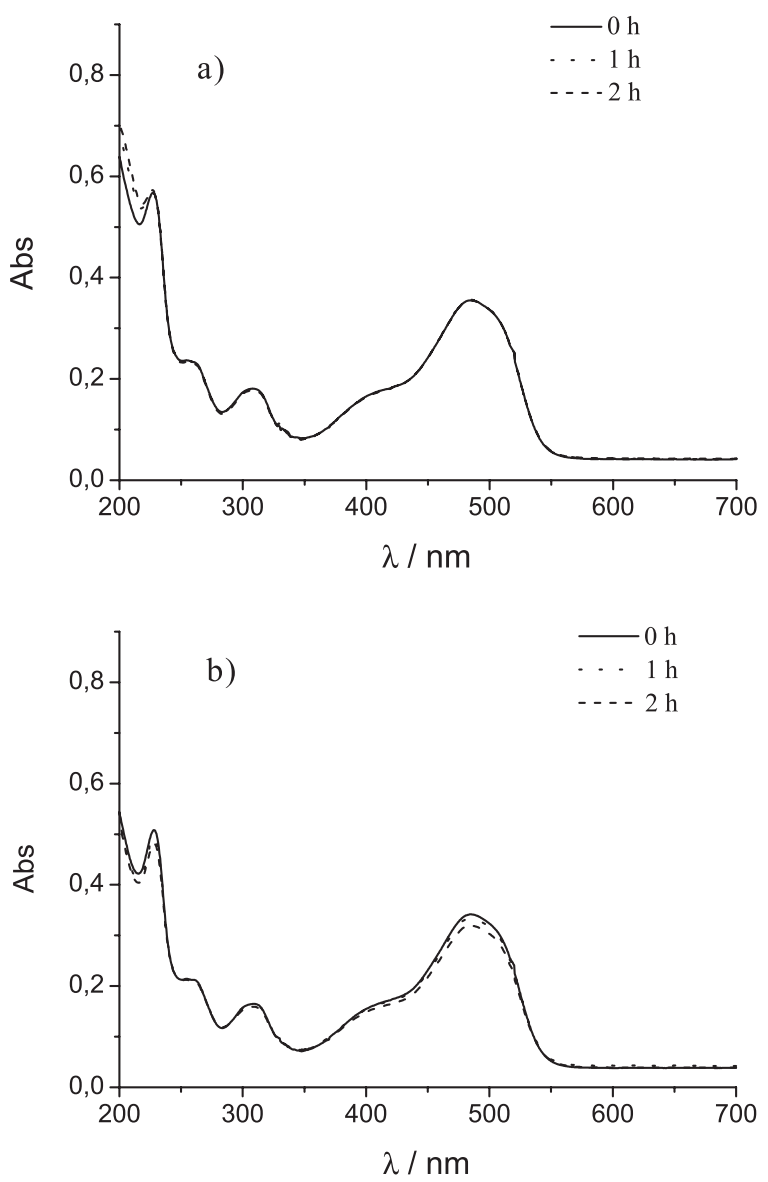

Figura 3. Evolução dos espetros de UV-Vis com o tempo de irradiação para

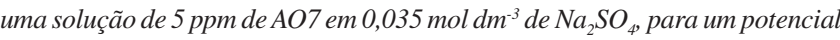
aplicado de $0,8 \mathrm{~V} v \mathrm{~s} \mathrm{~g} / \mathrm{AgCl}$. a) $\lambda=365 \mathrm{~nm}$ e b) luz branca

A Figura 3a mostra que não ocorre degradação significativa do corante $\mathrm{AO}$, pois não se observa a diminuição das bandas de absorção a 485, 310 e $228 \mathrm{~nm}$, características deste corante. No entanto, quando o fotoânodo é irradiado com luz branca, verifica-se uma ligeira diminuição das bandas de absorção a 485 e $228 \mathrm{~nm}$. Este resultado permite afirmar que ocorreu uma pequena degradação do corante. Para se obter a degradação total, seria necessário aumentar o tempo de irradiação mantendo o mesmo valor de potencial aplicado ou aplicar um potencial mais positivo.

Na Figura 4 está representada a evolução do espectro de UV-Vis da solução eletrolítica contendo AO7, quando se aplica um potencial de $1,0 \mathrm{~V}$ vs $\mathrm{Ag} / \mathrm{AgCl}$ sob irradiação de luz branca. Como se pode verificar, ocorreu uma diminuição considerável das bandas de absorção caraterísticas do corante, ao longo do tempo, comprovando a sua degradação parcial. A diminuição da banda de absorção a 485 $n m$ corresponde à quebra da ligação azo $(-\mathrm{N}=\mathrm{N}-)$ do corante. Estes resultados estão de acordo com os publicados para a degradação eletroquímica e fotoeletroquímica do $\mathrm{AO} 7$, usando eletrodos de diamante dopado com boro (BDD) e filmes mesoporosos de $\mathrm{TiO}_{2}$, respectivamente. ${ }^{35,45}$

A partir do valor de absorvância da banda a $485 \mathrm{~nm}$ foram calculadas as concentrações das amostras de $\mathrm{AO} 7$ ao longo do tempo de eletrólise, a partir da lei de Lambert-Beer. ${ }^{46}$ Através destes resultados foi possível calcular a percentagem de remoção de cor do corante, em 


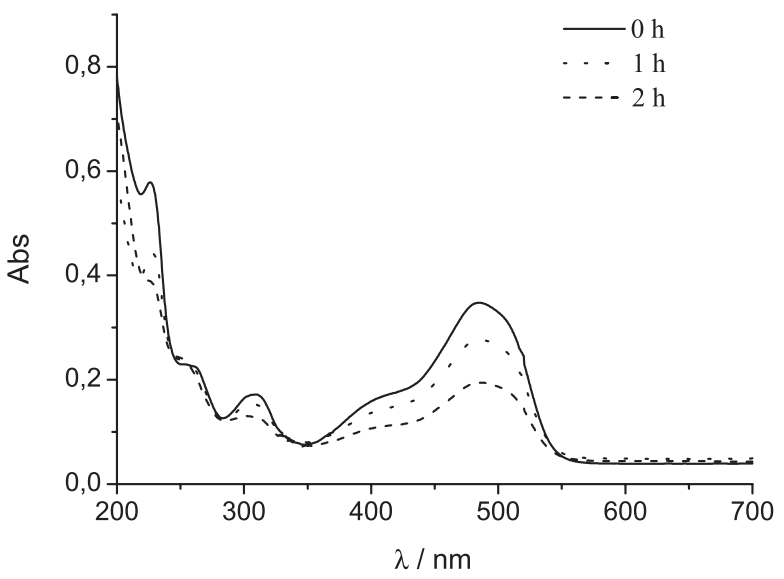

Figura 4. Evolução dos espetros de UV-Vis com o tempo de irradiação para uma solução inicial de 5 ppm de AO7 em 0,035 mol dm $\mathrm{me} \mathrm{Na}_{2}^{-3} \mathrm{SO}_{4}$, para um potencial aplicado de 1,0 Vvs Ag/AgCl e irradiação de luz branca

solução, ao longo da fotoeletrodegradação, Figura 5. Observa-se que ao fim de $2 \mathrm{~h}$ de ensaio, a percentagem de remoção de cor é aproximadamente $40 \%$. Hou et al. ${ }^{47}$ realizaram um estudo semelhante utilizando nanotubos de $\mathrm{TiO}_{2}$ na fotoeletrodegradação do $\mathrm{AO} 7$, obtendo $78 \%$ de degradação em $3 \mathrm{~h}$ para uma área geométrica de fotoânodo de $8 \mathrm{~cm}^{2}$. Considerando que a área geométrica dos filmes nanocompósitos é 10 vezes menor, pode-se concluir que a eletrodegradação fotoassistida do corante $\mathrm{AO} 7$ foi bem sucedida para o tipo de material estudado. A variação de $\ln \left(\mathrm{c} / \mathrm{c}_{0}\right)$ com o tempo de fotoeletrodegradação $(\mathrm{t})$ é linear. A constante aparente de velocidade de primeira ordem da reação de degradação pode ser estimada a partir da Equação 2:

$$
\ln \left(\frac{C}{C_{0}}\right)=-k t
$$

em que $c$ é a concentração de AO7 remanescente na solução no instante $t$ e $\mathrm{c}_{0}$ é a concentração de corante inicial a $t=0$.

$\mathrm{O}$ valor obtido para $k$ é de $4,12 \times 10^{-3} \mathrm{~min}^{-1}\left(0,247 \mathrm{~h}^{-1}\right)$, valor muito próximo ao obtido por Hou et al..$^{47}$ para a degradação deste corante utilizando filmes de $\mathrm{TiO}_{2}$.

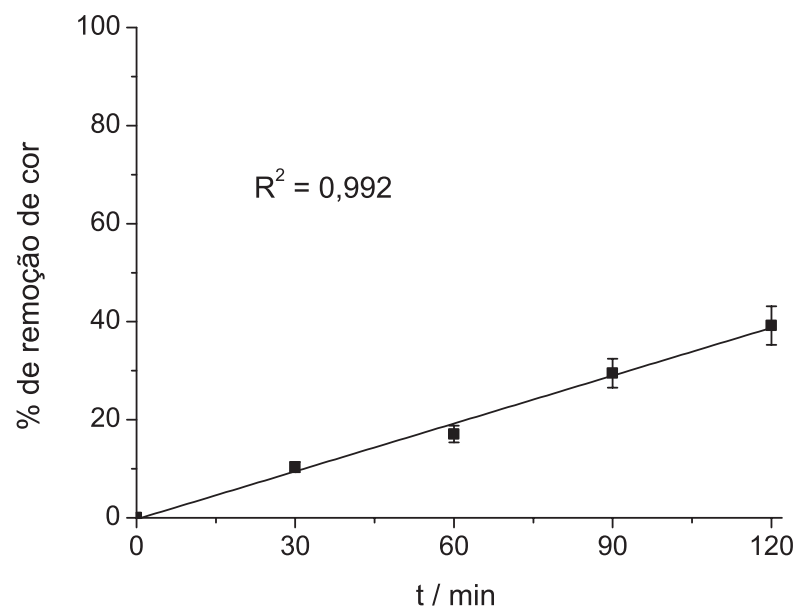

Figura 5. Variação da percentagem de remoção de cor de uma solução de 5 ppm de corante AO7 ao longo do tempo de fotoeletrodegradação

\section{CONCLUSÃO}

A preparação e utilização pela primeira vez de nanocompósitos de $\mathrm{Zn}-\mathrm{TiO}_{2}$ na eletrodegradação fotoassistida do corante $\mathrm{AO} 7$ foi realizada com sucesso. A análise de DRX dos nanocompósitos de $\mathrm{Zn}-\mathrm{TiO}_{2}$ confirma a conversão da matriz metálica de $\mathrm{Zn}$ para $\mathrm{ZnO}$ após o tratamento térmico, e os resultados de SEM indicam que os filmes apresentam uma elevada área com uma morfologia rica em nanoagulhas de $\mathrm{ZnO}$.

Os testes de fotoeletrodegradação realizados ao potencial de $1,0 \mathrm{~V}$ vs $\mathrm{Ag} / \mathrm{AgCl}$, em $0,035 \mathrm{~mol} \mathrm{dm}^{-3} \mathrm{Na}_{2} \mathrm{SO}_{4}$, com 5 ppm de AO7, sob irradiação de luz branca, durante $2 \mathrm{~h}$, demonstraram que os eletrodos utilizados são eficazes, tendo sido removida $40 \%$ da cor, sendo a constante aparente de velocidade de primeira ordem da reação de fotoeletrodegradação de $4,12 \times 10^{-3} \mathrm{~min}^{-1}$.

\section{AGRADECIMENTOS}

Ao financiamento da Fundação para a Ciência e Tecnologia (Portugal), sob o projeto de investigação PTDC/CTM/64856/2006.

\section{REFERÊNCIAS}

1. Hassemer, M. E. N.; Sens, M. L.; Engenharia Sanitária e Ambiental 2002, 7, 30 .

2. Oliveira, L. C. A.; Gonçalves, M.; Oliveira, D. Q. L.; Guerreiro, M. C.; Guilherme, L. R. G.; Dallago, R. M.; J. Hazard. Mater. 2007, 141, 344.

3. Méndez-Paz, D.; Omil, F.; Lema, J. M.; Enzyme Microb. Technol. 2005, 36, 264.

4. Kunz, A.; Zamora-Peralta, P.; Moraes, S. G.; Durán, N.; Quim. Nova 2002, 25, 78.

5. Anotai, J.; Lu, M.-C.; Chewpreecha, P.; Water Res. 2006, 40, 1841.

6. Huber, M. M.; Canonica, S.; Park, G.-Y.; Gunten, U. V.; Environ. Sci. Technol. 2003, 37, 1016.

7. Saritha, P.; Aparna, C.; Himabindu, V.; Anjaneyulu, Y.; J. Hazard. Mater. 2007, 149, 609.

8. Neyens, E.; Baeyens, J.; J. Hazard. Mater. 2003, 98, 33.

9. Rajkumar, D.; Palanivelu, K.; J. Hazard. Mater. 2004, B113, 123.

10. Méndez-Arriaga, F.; Esplugas, S.; Giménez, J.; Water Res. 2010, 44, 589.

11. Klauson, D.; Babkina, J.; Stepanova, K.; Krichevskaya, M.; Preis, S.; Catal. Today 2010, 15, 139.

12. Waldner, G.; Pourmodjib, M.; Bauer, R.; Neumann-Spallart, M.; Chemosphere 2003, 50, 989.

13. Zhao, X.; Qu, J.; Liu, H.; Qiang, Z.; Liu, R.; Hu, C.; Appl. Catal., B 2009, 91, 539 .

14. Antoniadou, M.; Kondarides, D. I.; Labou, D.; Neophytides, S.; Lianos, P.; Sol. Energy Mater. Sol. Cells 2010, 94, 592.

15. Mills, A.; Le Hunte, S.; J. Photochem. Photobiol., A 1997, 108, 1.

16. Fujishima, A.; Zhang, X.; Tryk, D. A.; J. Hydrog. Ener. 2007, 31, 2664.

17. Herrmann, J. M.; Catal. Today 1999, 53, 115.

18. Bahnemann, D.; Sol. Energy 2004, 77, 445.

19. Zhao, J.; Yang, X. D.; Build. Environ. 2003, 38, 645.

20. Khaselev, O.; Turner, J. A.; Science 1998, 280, 425.

21. Kondarides, D. I.; Daskalaki, V. M.; Patsoura, A.; Verykios, X. E.; Catal. Lett. 2008, 122, 26.

22. Graetzel, M.; Nature 2001, 414, 338.

23. Martínez-Huitle, C. A.; Brillas, E.; Appl. Catal., B 2009, 87, 105.

24. Rauf, M. A.; Ashraf, S. S.; Chem. Eng. J. 2009, 151, 10.

25. Pelton, R.; Geng, X.; Brook, M.; Adv. Colloid Interface Sci. 2006, 127, 43.

26. Mahmoodi, N. M.; Arami, M.; Limaee, N. Y.; J. Colloid Interface Sci. 2006, 295, 159.

27. Yue, B.; Zhou, Y.; Xu, J.; Wu, Z.; Zhang, X.; Zou, Y.; Jin, S.; Environ. Sci. Technol. 2002, 36, 1325.

28. Yassitepe, E.; Yatmaz, H. C.; Öztürk, C.; Duran, C.; J. Photochem. Photobiol., A 2008, 198, 1. 
29. Li, X. Z.; He, C.; Graham, N.; Ziong, Y.; J. Appl. Electrochem. 2005, 35, 741.

30. Cheng, S. T.; Chenthamarakshan, C. R.; Tacconi, N. R. de; Rajeshwar, K.; Res. Chem. Intermed. 2007, 33, 225.

31. Tacconi, N. R. de; Boyles, C. A.; Rajeshwar, K.; Langmuir 2000, 16, 5665.

32. Hoffmann, M. R.; Martin, S. T.; Choi, W. Y.; Bahnemann, D. W.; Chem. Rev. 1995, 95, 69.

33. Liu, R.; Ye, H.; Xiong, X.; Liu, H.; Mater. Chem. Phys. 2010, 121, 432.

34. Yuvaraj, D.; Rao, K. N.; Mater. Sci. Eng., B 2009, 164, 195.

35. Fernandes, A.; Morão, A.; Magrinho, M.; Lopes, A.; Gonçalves, I.; Dyes Pigm. 2004, 61, 287.

36. JCPDS-ICDD International Center for Diffraction Data; Powder Diffraction File Alphabetical Index, Swathmore: PA, 1988, File 4-0831.

37. Em ref. 36, File 36-1451.

38. Frade, T.; Bouzón, V.; Gomes, A.; Silva Pereira, M. I. da; Surf. Coat. Technol. 2010, 204, 3592
39. Deguchi, T.; Imai, K.; Iwasaki, M.; Tada, H.; Ito, S.; J. Electrochem. Soc. 2000, 147, 2263 .

40. Lin, C. S.; Lee, C. Y.; Chang, C. F.; Chang, C. H.; Surf. Coat. Technol. 2006, 200, 3690 .

41. Aal, A. A.; Mahmoud, S. A.; Aboul-Gheit, A. K.; Mater. Sci. Eng., C 2009, 29, 831.

42. Yu, W.; Pan, C.; Mater. Chem. Phys. 2009, 115, 74.

43. Yuvaraj, D.; Rao, K. N.; Mater. Sci. Eng., B 2009, 164, 195.

44. Yan, H.; Hou, J.; Fu, Z.; Yang, B.; Yang, P.; Liu, K.; Wen, M.; Chen, Y.; Fu, S.; Li, F.; Mater. Res. Bull. 2009, 44, 1954.

45. Carneiro, P. A.; Osugi, M. E.; Sene, J. J.; Anderson, M. A.; Zanoni, M. V. B.; Electrochim. Acta 2004, 49, 3807.

46. Skoog, D. A.; Holler, F. J.; Crouch, S. R.; Principles of intrumental analysis, Brooks/Cole: New York, 2006.

47. Hou, Y.; Li, X.; Liu, P.; Zou, X.; Chen, G.; Yue, P.-L.; Sep. Purif. Technol. 2009, 67, 135. 